\title{
Perception and Barriers to Indoor Air Quality and Perceived Impact on Respiratory Health: An Assessment in Rural Honduras
}

\author{
Audrey Le, ${ }^{1}$ Gonzalo Bearman, ${ }^{2}$ Kakotan Sanogo, ${ }^{3}$ and Michael P. Stevens ${ }^{2}$ \\ ${ }^{1}$ VCU School of Medicine, 1201 East Marshall Street, Richmond, VA 23298, USA \\ ${ }^{2}$ Department of Internal Medicine, VCU Medical Center, 1300 East Marshall Street, P.O. Box 980019, Richmond, VA, USA \\ ${ }^{3}$ VCUHS Infection Prevention Program, 1300 East Marshall Street, Richmond, VA 23298, USA
}

Correspondence should be addressed to Audrey Le; lea2@vcu.edu

Received 19 June 2014; Accepted 8 September 2014; Published 13 October 2014

Academic Editor: Ronald J. Prineas

Copyright (C) 2014 Audrey Le et al. This is an open access article distributed under the Creative Commons Attribution License, which permits unrestricted use, distribution, and reproduction in any medium, provided the original work is properly cited.

Objective. The aim of this study was to identify household-specific factors associated with respiratory symptoms and to study the perceived impact of indoor air pollution (IAP) as a health issue. Methods. An IRB-approved, voluntary, anonymous 23-item survey was conducted in Spanish at a medical outreach clinic in June 2012 and at the homes of survey respondents $(N=79)$. Comparative analyses were performed to investigate relationships between specific house characteristics and respiratory complaints. Results. Seventy-nine surveys were completed. Respiratory symptoms were frequently reported by survey respondents: $42 \%$ stated that smoke in their household caused them to have watery eyes, $42 \%$ reported household members with coughs within the past two weeks, and $25 \%$ stated that there were currently household members experiencing difficulty in breathing. Stove location and kitchen roof construction material were significantly associated with frequency of respiratory symptoms. The vast majority used firewood as their major fuel type. Most respondents indicated that neither indoor air quality was a problem nor did it affect their daily life. Conclusions. Respiratory complaints are common in Yoro, Honduras. Stove location and kitchen roof construction material were significantly associated with frequency of respiratory symptoms; this may have implications for efforts to improve respiratory health in the region.

\section{Introduction}

Approximately two-thirds of the developing world population or 3 billion people rely on biofuels such as wood, dung, and crop residues, for domestic energy [1]. Studies have shown that fuels used in simple household stoves produce significant amounts of respiratory pollutants and most notably substances such as polyaromatic compounds, particulate matter, formaldehyde, benzene, and 1,3-butadiene [2]. The level of exposure to these pollutants is many times higher for those who live in houses with limited ventilation compared to those whose homes meet the standards outlined by the World Health Organization Guidelines [2]. Indoor air pollution (IAP) increases the risk of pneumonia, chronic obstructive lung disease, and many other health conditions [1]. It is estimated that indoor air pollution is the cause of as many as 1.6-2 million deaths per year through its effect on respiratory illness as well as $20 \%$ of childhood deaths $(<5$ years old) per year due to acute lower respiratory infections [3]. Children aged 3 years and younger exposed to open-fire cooking are at greater risk than older children, likely due to more time spent around the home where open fires burn [4]. It may also be due to this particular time period when changes to the lungs and other organs, including the brain, are most susceptible. Recently, Munroe and Gauvain showed that children who are exposed to open-fire cooking showed lower cognitive performance and less frequent structured play compared to those exposed to kerosene stoves [4].

A prior needs assessment survey that included people in La Hicaca, Honduras, reported that approximately $60 \%$ of respondents used indoor household stoves to do their daily cooking and that over $70 \%$ used fire wood as their primary 
cooking fuel. The survey also identified indoor air quality as a major health concern (Stevens M.P. et al., 2009, unpublished data).

Studies in Latin American and Asian populations have reported failure to adopt improved stoves even following extensive efforts to promote their usage [5]. This is thought to be due to a variety of factors, including cost, inconvenience, and inability to accommodate meal preparation traditions [5]. Efforts to promote the adoption of cleaner fuels have also proven to be futile; in many places, they are not available, and, in areas in which they are, there is a failure to adopt cleaner fuels mainly due to the cheaper cost of biomass fuels [5]. Thus, such studies suggest that, in order to improve indoor air quality in rural, impoverished households, alternative solutions are necessary. Several studies have shown that specific housing characteristics such as kitchen location, ventilation (chimneys, area of windows, doors, etc.), and construction material can play an important role in IAP $[5,6]$.

Since 2008, Virginia Commonwealth University's Global Health and Health Disparities Program (GH2DP) has been providing medical and public health services in and around the rural, remote village of La Hicaca, Department of Yoro, Honduras. IAP was identified as a concern in the region by GH2DP's community partners and this study was designed to formally assess this issue. The main aim of this study was to identify household-specific factors associated with respiratory symptoms and to study the perceived impact of indoor air pollution as a health issue in a rural Honduran community.

\section{Methods}

An anonymous, voluntary 23-item questionnaire was approved by the Institutional Review Board of Virginia Commonwealth University. This survey tool was employed at the point of care in the GH2DP clinic site and at the homes of survey respondents in June 2012. The survey was written in English, then translated to Spanish, and then back-translated to English for accuracy. The translation was performed by native Spanish speakers (Appendix A available online in supplementary material at http://dx.doi.org/10.1155/2014/105260). The survey was adopted and modified from a previously written questionnaire [7]. Written permission from the original author was obtained. The survey had two components. The first component aimed to identify determinants of indoor air pollution through multiple choice questions about fuel, stove type, construction material of kitchen roofs, walls, and floors, number of windows/doors in the kitchen, and presence of chimney within the home. The second portion focused on self-reported respiratory complaints such as number of respondents and their household members experiencing cough within the past two weeks and experiencing difficulty in breathing, currently being treated for respiratory illness, and household deaths due to respiratory problems. These questions were multiple choice, yes/no-type, or on a 3-item scale; for example, one question evaluated severity of cough by having "mild," "moderate," and "severe" as possible answer choices.
TABLE 1: Survey respondents and village of origin.

\begin{tabular}{lcc}
\hline Village & $\begin{array}{c}\text { Number of } \\
\text { respondents from } \\
\text { village }\end{array}$ & $\begin{array}{c}\text { Total number of } \\
\text { households in village }\end{array}$ \\
\hline El Portillo & $5(6 \%)$ & 53 \\
San Felix & $3(4 \%)$ & 4 \\
La Esperanza & $4(5 \%)$ & 9 \\
La Florida & $3(4 \%)$ & 18 \\
Santa Maria & $2(2 \%)$ & 28 \\
Agua Sarca & $5(6 \%)$ & 33 \\
La Culata & $3(4 \%)$ & 9 \\
La Hicaca & $39(49 \%)$ & 11 \\
Lomitas & $4(5 \%)$ & 15 \\
El Urraco & $2(2 \%)$ & 6 \\
Puerto Rico & $1(1 \%)$ & 14 \\
Agua Caliente & $6(7 \%)$ & 4 \\
La Vega & $1(1 \%)$ & 3 \\
La Lima & $1(1 \%)$ &
\end{tabular}

An additional question asked about the perceived impact of indoor air quality on daily life, with responses such as "not a problem that affects my daily life," "a minor problem that somewhat affects my daily life," "a major problem that inhibits my daily life," or "not sure."

Eligible subjects included adults above 18 years of age. The study was conducted over a five-day span. All interviews were conducted in Spanish with the first author in attendance. Survey results were recorded on paper and then transferred to a password-protected Microsoft Excel spreadsheet database.

Statistical Analysis Software (SAS, version 9.2, SAS Institute Inc., Cary, NC, USA) was used to perform descriptive analyses of data via frequency counts of responses. Subsequently, comparative analyses were performed to investigate the relationship between specific house characteristics and respiratory complaints. A chi-squared test was used to evaluate the significance of differences between response groups.

A schematic diagram of stove locations was included in the survey questionnaire (Appendix A). Stove locations 1, 2, and 3 were grouped together as "indoor stoves," Stoves $4 \mathrm{~A}$ and $4 \mathrm{~B}$ were grouped together as "attached stoves," and Stoves 5 and 6 were grouped together as "outdoor stoves."

Respiratory symptom responses were analyzed as twoway comparisons by presence of chimney and by roof type. A three-way test comparison was employed to assess respiratory symptom responses by stove location.

IRB title and number are as follows: Assessing Indoor Air Quality in Rural Honduras-the VCU Global Health and Health Disparities Program, HM14252.

\section{Results}

Seventy-nine surveys were obtained. The mean number of people living per household was 6 (range: 1-12 persons). Forty-nine percent $(39 / 79)$ of the survey respondents were from La Hicaca and 51\% (40/79) were from surrounding 
TABLE 2: Summary of common house construction materials. Total $N=79$.

\begin{tabular}{lcc}
\hline $\begin{array}{l}\text { House } \\
\text { characteristic }\end{array}$ & Major material used & Number of households \\
\hline Roof & Corrugated zinc & $48(61 \%)$ \\
Walls & Adobe/mud & $63(80 \%)$ \\
Floor & Mud & $66(84 \%)$ \\
Stove & Mud/adobe/sand & $74(94 \%)$ \\
Fuel & Firewood & $76(96 \%)$ \\
Chimney & - & $61(77 \%)$ \\
\hline
\end{tabular}

villages (Table 1). House characteristics are summarized in Table 2. Ninety-six percent (76/79) reported firewood as their major fuel type and 77\% (61/79) reported chimneys within their homes. Figure 1 summarizes stove locations identified by respondents; thirty-seven percent (29/79) reported indoor stove locations, $20 \%$ (16/79) outdoor stoves, and 40\% (32/79) attached stoves. Sixty-one percent (48/79) of respondents had kitchen roofs constructed of corrugated zinc, $80 \%$ (63/79) had walls of mud/adobe, $84 \%(66 / 79)$ had mud floors within their kitchen, and 94\% (74/79) had stoves made of mud/adobe/sand (Table 2).

Eighty-one percent $(n=64)$ of respondents denied the presence of a smoker in the household. Six percent (5/79) identified IAP as a health concern; eighty-nine percent (70/79) of respondents indicated that it was not a problem that affected their daily life. Figure 2 summarizes reports of respiratory symptoms. Twenty-seven percent (21/79) of survey respondents reported one or more household members currently being treated for respiratory problems, $42 \%$ $(33 / 79)$ stated that household smoke resulted in watery eyes, and $42 \%(33 / 79)$ reported household members with cough over the past two weeks. Twenty-five percent (20/79) of survey participants stated that there were currently household members experiencing difficulty in breathing. Eight percent (6/79) of survey respondents reported deaths within their household due to respiratory illness, with 50\% (3/6) occurring in children less than 5 years of age.

Table 3 summarizes the comparison of respiratory symptoms by house characteristics. No differences in respiratory symptoms were observed by the presence of a chimney. Corrugated roofs compared to other roof types such as tile, thatched, and concrete roofs were associated with the fewest reports of family members being treated for respiratory symptoms. Outdoor stoves were associated with increased reports of watery eyes due to smoke and family members being treated for respiratory problems compared to attached stove locations (Figure 3).

Eighty-eight percent $(n=70)$ of respondents did not believe that IAP was a health problem (Figure 4).

\section{Discussion and Conclusions}

This study focused on perceptions and barriers to indoor air quality in rural Honduras. The data demonstrate nearly uniform usage of stove and fuel types; $96 \%$ of respondents used firewood as their main fuel type and $94 \%$ used mud/adobe/sand stoves. Despite not being perceived as a major health concern, respiratory symptoms were commonly reported.

We found that the attached stove location was associated with fewer respiratory complaints when compared to the indoor and outdoor stove locations. We postulated that the attached model more efficiently reduces the exposure to smoke over the outdoor stove. As rural Hondurans spend a lot of time congregating outside of their homes, an outdoor stove in close proximity may expose them to a large amount of smoke. In contrast, an attached stove may serve to better distance household members from the pollutants generated by cooking. However, when stove location was statistically adjusted for by the presence or absence of a chimney, the difference in respiratory symptoms was no longer significant (see Appendix B, Table B-1).

IAP from biomass fuels has long been regarded as a major risk factor for acute respiratory illness. Acute respiratory illness is the second leading cause of death in children aged 5 years and under globally [8]. Interestingly, despite the high frequency of reported respiratory complaints, IAP was not perceived as a major health issue by $89 \%$ of study participants. Results from prior surveys indicate that the majority of people in the region have received a 6th grade education or less (Stevens M.P. et al., 2009, unpublished data). These data suggest that an IAP education gap exists. The resource-poor inhabitants of La Hicaca and the surrounding villages may not perceive respiratory health as a pressing matter when other concerns related to subsistence living exist. Similar findings have been reported in other rural communities. Diaz et al. similarly studied IAP and self-reported health symptoms amongst Mayan women. Although survey respondents suffered daily from symptoms of headache and eye discomfort, their perception of personal health was positive [9]. Angel and Thoits theorize that the more prevalent an objectively abnormal physical or emotional condition is in one's reference group, the less likely one is to attribute great significance to it [10]. Under this framework, the high prevalence of self-reported respiratory conditions may be perceived as a norm by our study population. Further, this finding underscores the critical importance of education about the detrimental effects of IAP in order to affect change. Thus, education on IAP and improvement in cook stoves, in collaboration with the local Ministry of Health, are a future mission of the GH2DP medical relief team.

This study had several strengths. Multiple ventilation factors that could potentially impact respiratory health were evaluated, such as number of windows, stove location, and roofing material. All interviews were done in Spanish with a structured questionnaire. The majority of individuals invited to participate agreed. The interviewers traveled daily from house to house in order to interview inhabitants of La Hicaca, the largest village within the region, potentially decreasing the sampling bias of an exclusive clinic population.

Study limitations included the small sample size. Nearly half $(49 \%)$ of the interviews were conducted within La 


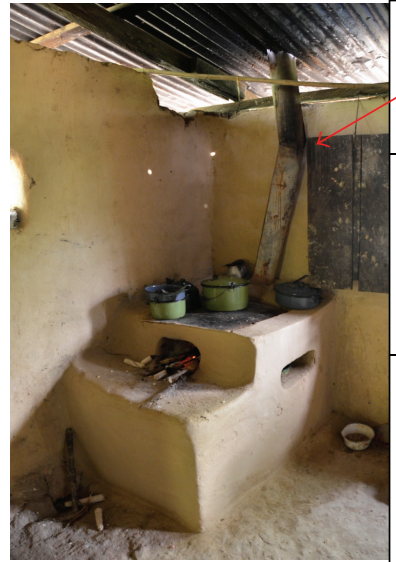

(a)

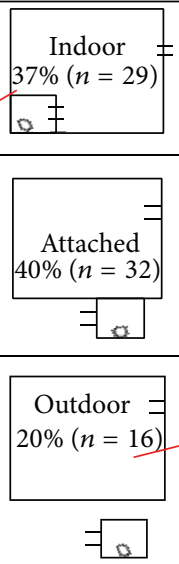

(b)

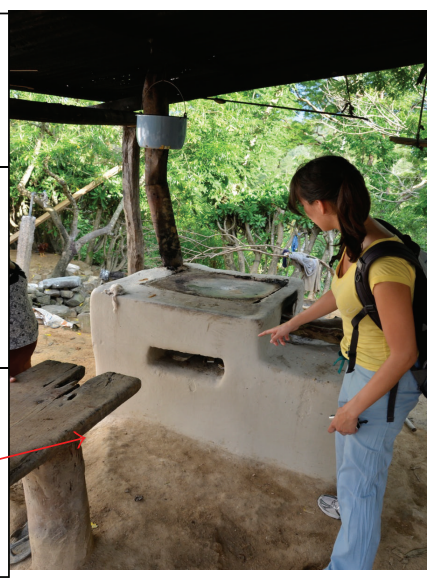

(c)

Figure 1: Diagram of stove locations. (b) Schematic diagram of stove locations (see Appendix A). Thirty-seven percent of individuals identified their stove in an indoor location, $40 \%$ identified their stove in an attached location (w.r.t. their main living quarters), and $20 \%$ identified their stove location in an outdoor location. (c) Typical outdoor stove. (a) Typical indoor stove.

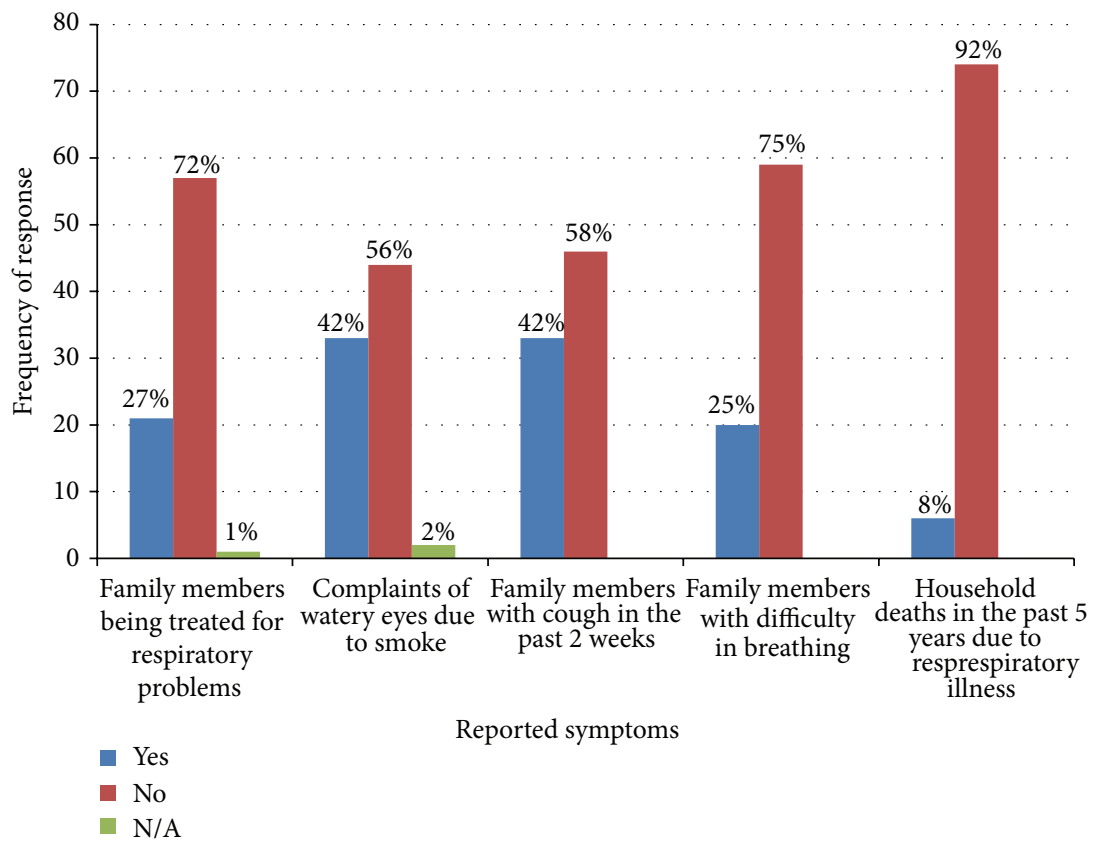

FIGURE 2: Summary of reported respiratory symptoms.

Hicaca, with the majority done during house-to-house visitations. Still, there remains the limitation of convenience sample methodology (with the attendant concern that participants from other villages may not truly represent the population in their region), as well as recall bias. No formal evaluation of the adequacy of chimney construction was performed.

To date, few studies have addressed IAP in rural Honduras. This study adds to the body of literature on respiratory health in Central America and Honduras. Clark et al. investigated IAP in the Honduran villages of Santa Lucia and Suyapa and demonstrated that improved stove usage is associated with $73 \%$ lower levels of indoor particulate matter and $87 \%$ lower levels of indoor carbon monoxide levels compared to traditional stoves [11]. Women who used traditional stoves reported symptoms such as cough, shortness of breath, and wheezing more frequently than those with improved stoves. In the Department of Yoro, usage of firewood and adobe mud stoves is nearly uniform. In our study we included house structural characteristics such as stove location and roofing material to investigate other potential contributors to respiratory symptoms. House characteristics are often overlooked as factors in IAP, when they may, in fact, play a critical role, especially in regions where biomass fuels cannot be easily replaced. Dasgupta et al. suggest that, in areas where adoption of clean fuel or improved stoves may 
TABLE 3: Comparison of respiratory symptoms by household characteristic.

\begin{tabular}{|c|c|c|c|c|c|c|c|}
\hline Symptom & $\begin{array}{l}\text { Chimney } \\
(N=61)\end{array}$ & $\begin{array}{l}\text { No chimney } \\
(N=16)\end{array}$ & $\begin{array}{c}\text { Indoor } \\
(N=29)\end{array}$ & $\begin{array}{l}\text { Outdoor } \\
(N=16)\end{array}$ & $\begin{array}{l}\text { Attached } \\
(N=31)\end{array}$ & $\begin{array}{c}\text { Corrugated zinc } \\
\quad(N=49)\end{array}$ & $\begin{array}{l}\text { Other roofs }{ }^{\dagger} \\
(N=28)\end{array}$ \\
\hline $\begin{array}{l}\text { Treated for } \\
\text { respiratory problems }\end{array}$ & $15(25 \%)$ & $5(31 \%)$ & $9(31 \%)$ & $7(44 \%)^{*}$ & $4(12 \%)^{*}$ & $9(18 \%)^{*}$ & $12(43 \%)^{*}$ \\
\hline $\begin{array}{l}\text { Watery eyes due to } \\
\text { household smoke }\end{array}$ & $25(41 \%)$ & $7(44 \%)$ & $11(38 \%)$ & $10(62 \%)^{*}$ & $11(34 \%)^{*}$ & $21(43 \%)$ & $12(43 \%)$ \\
\hline $\begin{array}{l}\text { Cough in the past two } \\
\text { weeks }\end{array}$ & $25(41 \%)$ & $7(44 \%)$ & $14(48 \%)$ & $8(50 \%)$ & $10(31 \%)$ & $20(41 \%)$ & $12(43 \%)$ \\
\hline Difficulty in breathing & $14(23 \%)$ & $5(31 \%)$ & $9(31 \%)$ & $6(38 \%)$ & $4(12 \%)$ & $10(20 \%)$ & $10(36 \%)$ \\
\hline $\begin{array}{l}\text { Deaths due to } \\
\text { respiratory problems }\end{array}$ & $3(5 \%)$ & $2(12 \%)$ & $1(4 \%)$ & $1(6 \%)$ & $3(9 \%)$ & $3(6 \%)$ & $3(11 \%)$ \\
\hline
\end{tabular}

From left to right: two-way test of statistical significance to compare frequency of reported respiratory symptoms by absence or presence of chimney, by stove location, and by roof material.

* denotes a paired $P$ value $<0.05$.

${ }^{\dagger}$ other roofing material included such types as adobe, mud, thatched, cement, tile, or a mixture of them.

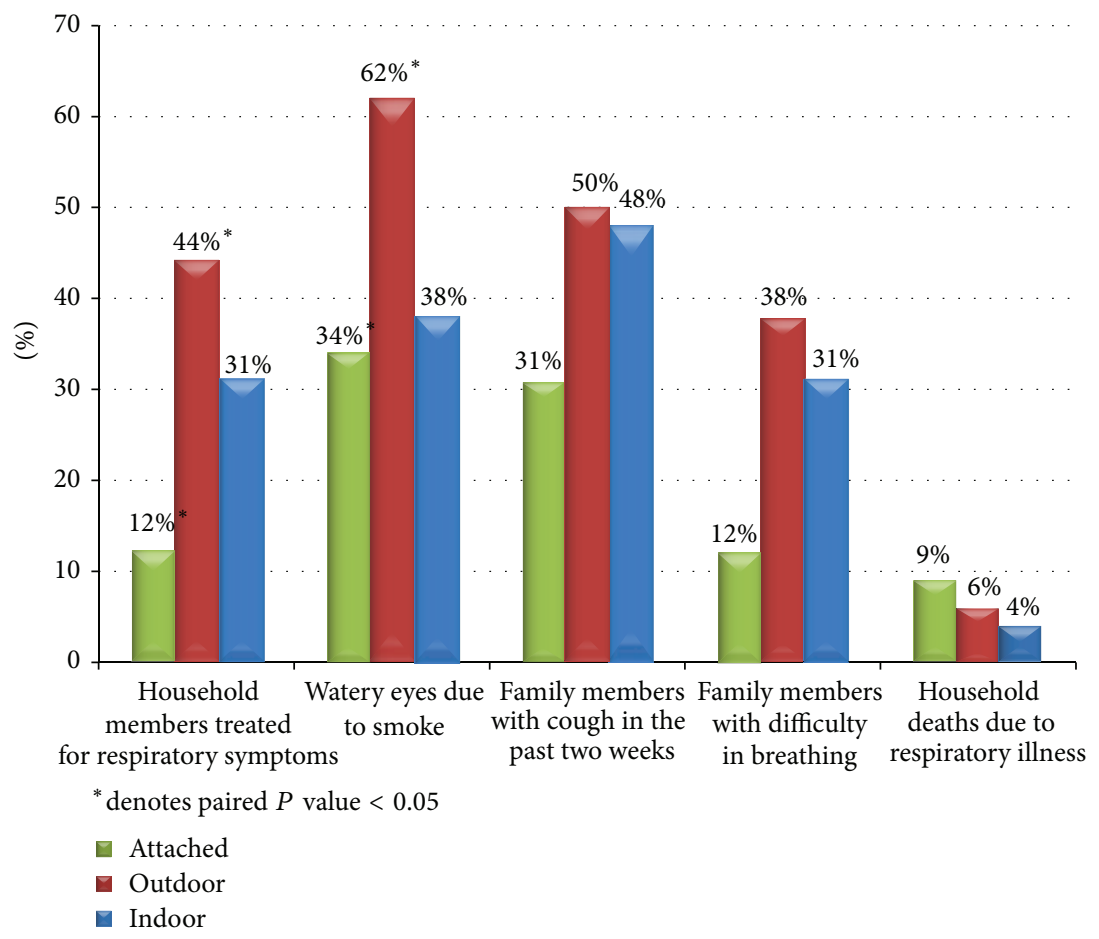

FIGURE 3: Comparison of respiratory symptoms by stove location.

not be feasible, changes in construction materials, space configurations, and cooking locations can be an alternative approach to improving overall respiratory health [5]. This study concluded that outdoor air pollution is a significant determinant of indoor ambient pollution, which would parallel our observation of increased respiratory complaints amongst those with outdoor stove locations (Figure 3). In addition, we observed that respondents whose homes had corrugated zinc roofs reported fewer respiratory complaints. We postulate that the more porous thatched or straw roofs could potentiate exchange of smoke between kitchen and the rest of the home, causing distribution of smoke throughout the house. One could also argue that a more porous roof would allow greater ventilation; thus, this requires further investigation. Dasgupta and colleagues determined tin roofs to be significantly better than thatch roofs within the kitchen, which would also be consistent with these findings.

This study's findings suggest a role for structural modification of homes to decrease respiratory symptoms in rural Honduras. Notably, one of the things we determined through formal discussions with our community partners after conducting this study was that many of the chimneys in La Hicaca are in disrepair and thus are likely not effectively ventilating smoke. The randomized controlled RESPIRE trial, 


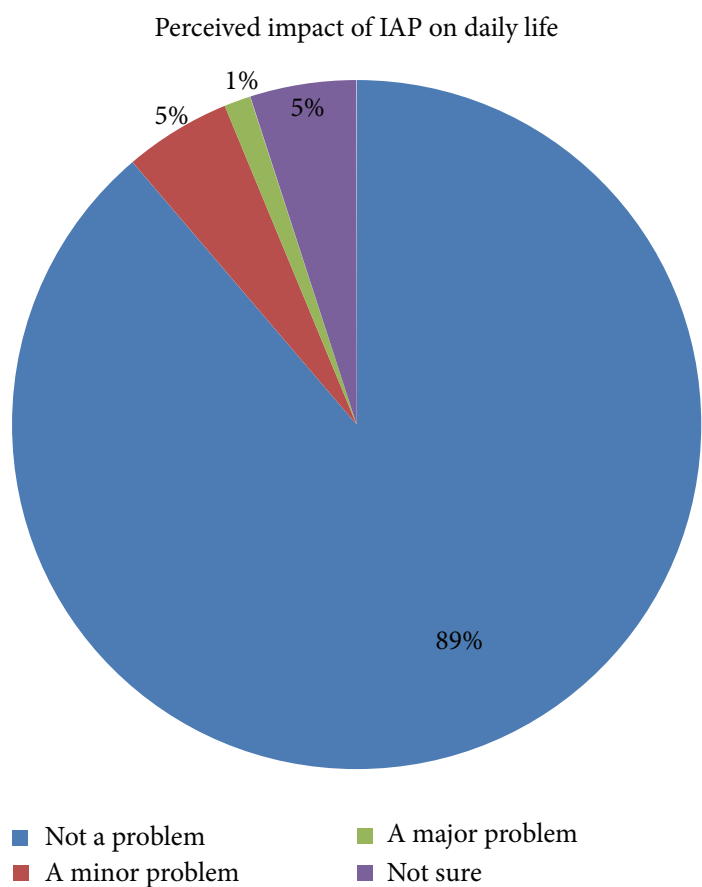

FIgUre 4: Perception of IAP as a health issue.

conducted by Smith et al., Guatemala, also demonstrated that there was no significant reduction in physician-diagnosed pneumonia in children living in homes with chimney stoves compared to those without chimneys [12]. The group found that, although kitchens with chimney stoves showed a reduction by a factor of 10 in mean concentrations of carbon monoxide, child exposures were only lowered by a factor of two. It was concluded that the difference was due to stoves simply venting smoke to the immediate outdoors, some of which reenters the homes [12]. This is an area for future study and intervention. Ongoing education on the negative impact of IAP on respiratory health is needed.

IAP is a continuous, widespread problem in rural Honduras. Although respiratory symptoms were common across all study participants, the perception of IAP as a health detriment was not widely recognized. Although fuel type did not vary across the study population, opportunities exist to improve cook stove location and to optimize the use of air ventilating chimneys. Household structural changes must be accompanied by ongoing educational efforts to recognize and underscore the negative impact of IAP on respiratory health.

\section{Disclaimer}

The views expressed in this submitted paper are those of the authors and not an official position of Virginia Commonwealth University.

\section{Conflict of Interests}

The authors declare that there is no conflict of interests regarding the publication of this paper.

\section{Acknowledgment}

The authors would like to thank Susmita Dasgupta, Ph.D. of the World Bank Organization holder, who granted them permission to modify the needs assessment survey underlying her research in Bangladesh for their own study's purposes $[3,5]$.

\section{References}

[1] N. Bruce, J. McCracken, R. Albalak et al., "Impact of improved stoves, house construction and child location on levels of indoor air pollution exposure in young Guatemalan children," Journal of Exposure Analysis \& Environmental Epidemiology, vol. 14, no. 1, pp. S26-S33, 2004.

[2] K. R. Smith and S. Mehta, "The burden of disease from indoor air pollution in developing countries: comparison of estimates," International Journal of Hygiene and Environmental Health, vol. 206, no. 4-5, pp. 279-289, 2003.

[3] S. Dasgupta, M. Huq, M. Khaliquzzaman, K. Pandey, and D. Wheeler, "Indoor air quality for poor families: new evidence from Bangladesh," Indoor Air, vol. 16, no. 6, pp. 426-444, 2006.

[4] R. L. Munroe and M. Gauvain, "Exposure to open-fire cooking and cognitive performance in children," International Journal of Environmental Health Research, vol. 22, no. 2, pp. 156-164, 2012.

[5] S. Dasgupta, D. Wheeler, M. Huq, and M. Khaliquzzaman, "Improving indoor air quality for poor families: a controlled experiment in Bangladesh," Indoor Air, vol. 19, no. 1, pp. 22-32, 2009.

[6] M. L. Clark, S. J. Reynolds, J. B. Burch, S. Conway, A. M. Bachand, and J. L. Peel, "Indoor air pollution, cookstove quality, and housing characteristics in two Honduran communities," Environmental Research, vol. 110, no. 1, pp. 12-18, 2010.

[7] S. Dasgupta, M. Huq, K. D. Pandey, M. Khaliquzzaman, and D. R. Wheeler, Exposure to Indoor Air Pollution in Bangladesh, The World Bank Group, 2004, http://econ.worldbank.org.

[8] A. Emmelin and S. Wall, "Indoor air pollution: a poverty-related cause of mortality among the children of the world," Chest, vol. 132, no. 5, pp. 1615-1623, 2007.

[9] E. Diaz, N. Bruce, D. Pope, A. Diaz, K. R. Smith, and T. SmithSivertsen, "Self-rated health among Mayan women participating in a randomised intervention trial reducing indoor air pollution in Guatemala," BMC International Health and Human Rights, vol. 8, article 7, 2008.

[10] R. Angel and P. Thoits, "The impact of culture on the cognitive structure of illness," Culture, Medicine and Psychiatry, vol. 11, no. 4, pp. 465-494, 1987.

[11] M. L. Clark, J. L. Peel, J. B. Burch et al., "Impact of improved cookstoves on indoor air pollution and adverse health effects among Honduran women," International Journal of Environmental Health Research, vol. 19, no. 5, pp. 357-368, 2009.

[12] K. R. Smith, J. P. McCracken, M. W. Weber et al., "Effect of reduction in household air pollution on childhood pneumonia in Guatemala (RESPIRE): a randomised controlled trial," The Lancet, vol. 378, no. 9804, pp. 1717-1726, 2011. 


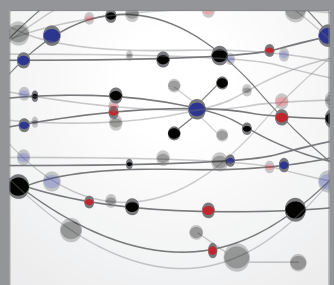

The Scientific World Journal
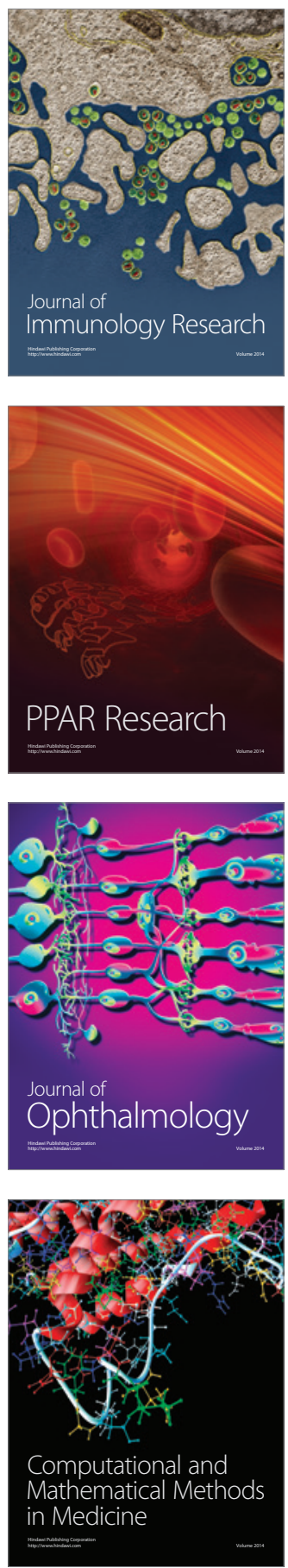

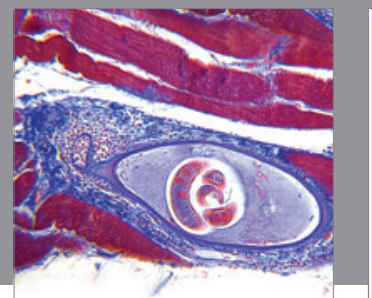

Gastroenterology

Research and Practice
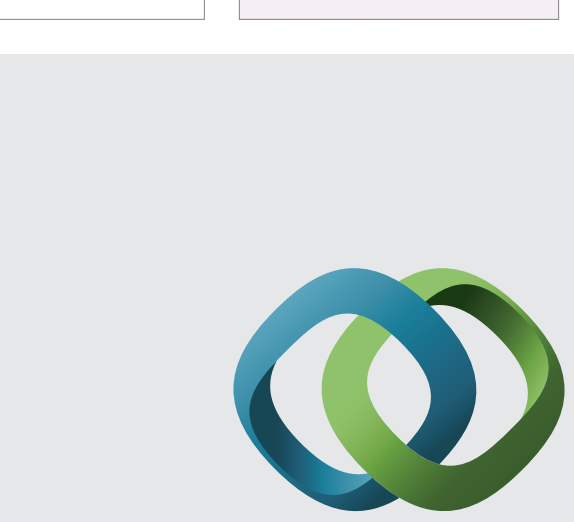

\section{Hindawi}

Submit your manuscripts at

http://www.hindawi.com
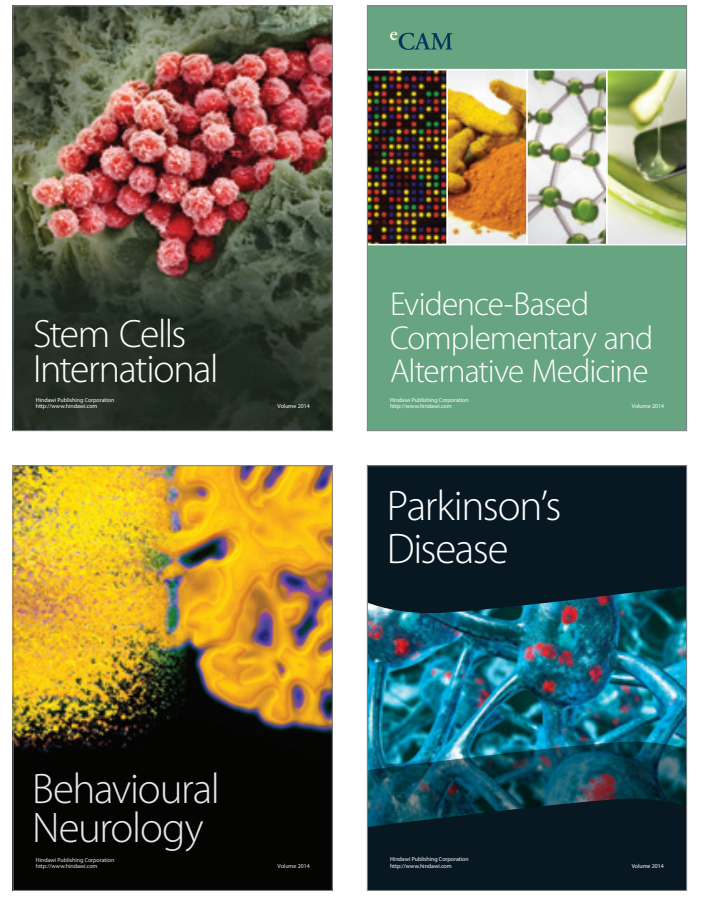
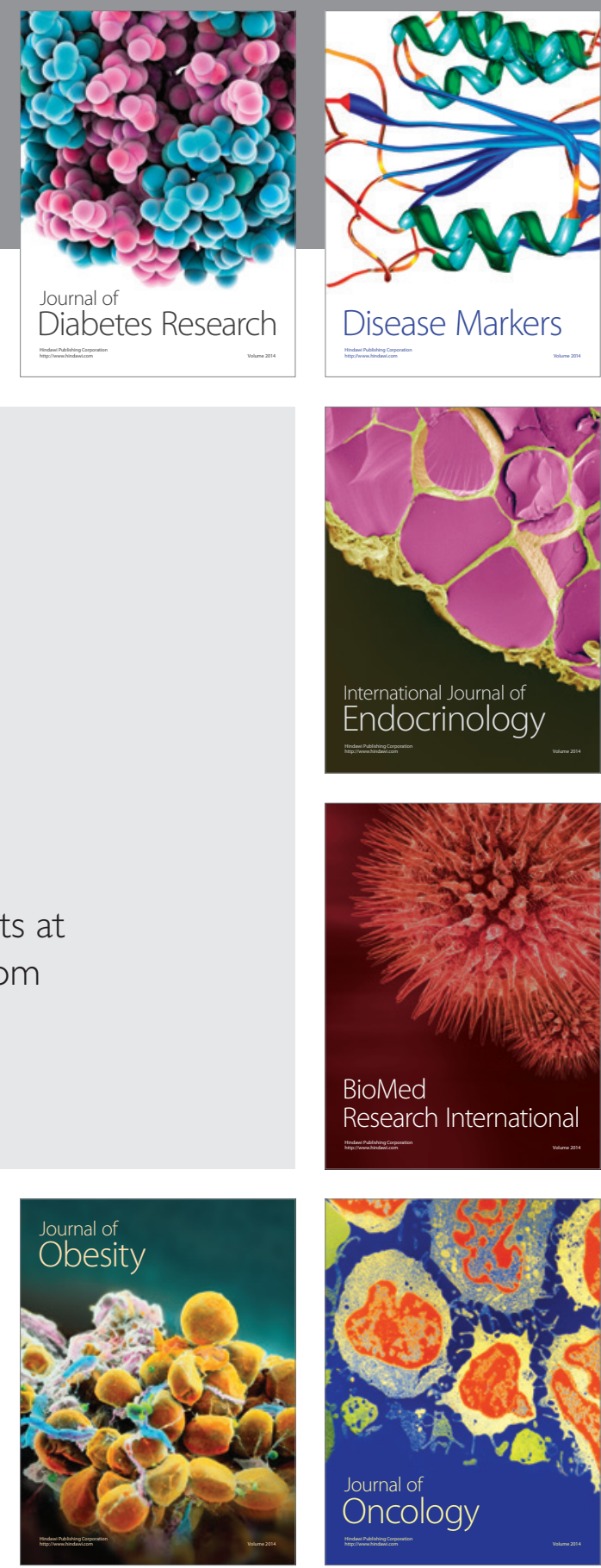

Disease Markers
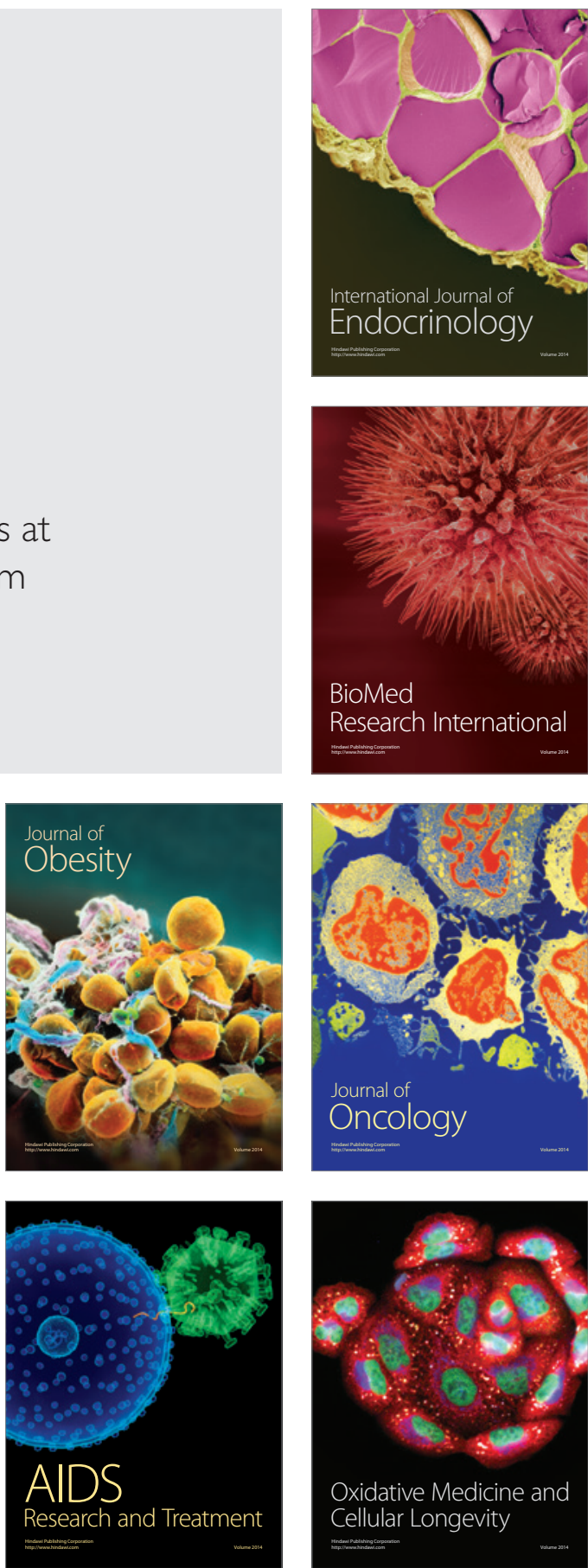